\title{
The Impact of Cylindrical Fuel Assemblies' Arrangement on the Heat and Mass Transfers into an Horizontal Porous Circular Cylinder
}

\author{
Karim RAGUI ${ }^{1, ~}{ }^{,}$, Abdelkader BOUTRA ${ }^{1,2}$ and Youb Khaled BENKAHLA ${ }^{1}$ \\ ${ }^{1}$ Laboratory of Transport Phenomena, USTHB, Algiers, Algeria. \\ ${ }^{2}$ Preparatory School of Science and Technology, Algiers, Algeria. \\ E-mail address: ragui-karim@live.fr, aeknad@yahoo.fr, youbenkahla@yahoo.fr
}

\begin{abstract}
Keywords: Computational fluid dynamic/ Heat and mass transfer/ Porous horizontal cylinder/ Cylindrical fuel assemblies/ Rectangular disposition/ Lozenge disposition/ Powerful correlations.
\end{abstract}

\begin{abstract}
The present work refers to the investigation of natural convection into an horizontal porous circular cylinder, driven by cooperating thermal and solutal buoyancy forces. The circular cylinder is maintained at constant temperature and concentration lower than that of four inner, heat and solute, ones which are arranged in two different manners. The physical model for the momentum conservation equation makes use of the Brinkman extension of the classical Darcy equation, the set of coupled equations is solved using the finite volume method and the SIMPLER algorithm. Through a parametric study, the effects of the distance between the cylinders on the thermal and solutal characteristics are widely inspected. Then, powerful correlations predicting the mean transfer inside the porous medium as a function of the cylinders' disposition are proposed, which predict within $\pm 1 \%$ the numerical results. It is to note that the validity of the computing code used was ascertained by comparing our results with the experimental and the numerical ones already available in the literature.
\end{abstract}

\section{INTRODUCTION}

Double-diffusive natural convection analysis into porous medium has been the subject of a very intense research activity over the past decades, due to the importance of related industrial and technological applications. Prominent among the latter being geothermal energy resources; nuclear energy systems; petroleum reservoirs; analysis of insulating systems; grain storage; pollutant dispersion in aquifers; the processes of crystal manufacture, foam metals and fibrous media; to name but a few. [1-3].

With both temperature and concentration gradients present to drive the fluid flow, an increased number of transport configurations is possible, with parallel or perpendicular gradients, and the body forces augmenting or opposing [4-10].

In the main idea to predict heat and mass transfer into such configurations, powerful correlations were proposed: In 1987, Trevisan and Bejan [11] projected the thermal and solutal transfer, quantified by Sherwood and Nusselt numbers, into a square porous medium as function of the thermal Rayleigh and the Lewis numbers, as well as the aspect and the buoyancy ratios.

In 1990 Lin et al. [12] proposed Nusselt and Sherwood correlations as function of the thermal Grashof number and that, only with small values of the buoyancy ratio $(|\mathrm{N}|<5)$.

In 1993, Bennacer [13] suggested a general correlation for mass transfer inside square enclosures, which may used in a wide range of the porous thermal Rayleigh number, the buoyancy ratio, and the Lewis number as well.

Motivated by these works, and by the fact that the investigation of double-diffusive convection, into such porous geometry as our, may less common in the available literature, this paper will discuss the results of the latter into a porous circular cylinder that contains four heat and solute cylinders, arranged in a rectangular or lozenge manner.

The purpose of this contribution is to show the impact of pertinent parameters, such the Lewis and the porous thermal Rayleigh numbers, the buoyancy ratio, as well as the cylinders' arrangement 
on the double-diffusive natural convection into such geometry, to come out at the end with global correlations predicting the mean heat and mass transfer and that, for the convenience of practical applications such as cooling fuel assemblies of nuclear core reactors, and predicting pollutants spreading into heat exchangers.

\section{PROBLEM STATEMENT AND MATHEMATICAL FORMULATION}

The studied configuration, shown in Fig. 1, consists of a cold (less concentric) porous circular cylinder containing four heat and solute cylinders mounted inside in a rectangle (Fig. 1(a)) or a lozenge arrangement (Fig. 1(b)). The fluid filled the porous medium is assumed to be Newtonian, its thermophysical properties are presumed to be constant except the density variation, in the buoyancy term, which depends linearly to the local temperature and concentration.

$$
\rho_{(\mathrm{T}, \mathrm{C})}=\rho_{0}\left[1-\beta_{\mathrm{T}}\left(\mathrm{T}-\mathrm{T}_{0}\right)-\beta_{\mathrm{C}}\left(\mathrm{C}-\mathrm{C}_{0}\right)\right]
$$

where $\beta_{\mathrm{T}}$ and $\beta_{\mathrm{C}}$ are the thermal and the solutal expansion coefficients:

$$
\beta_{\mathrm{T}}=-\frac{1}{\rho_{0}}\left(\frac{\partial \rho}{\partial \mathrm{T}}\right)_{\mathrm{p}}, \beta_{\mathrm{C}}=-\frac{1}{\rho_{0}}\left(\frac{\partial \rho}{\partial \mathrm{C}}\right)_{\mathrm{p}}
$$

The solid matrix is supposed to be isotropic, homogeneous and in thermal equilibrium with the fluid. The permeability of the porous medium $\mathrm{K}$ is kept uniform, when the porosity $\varepsilon$ is about $40 \%$.

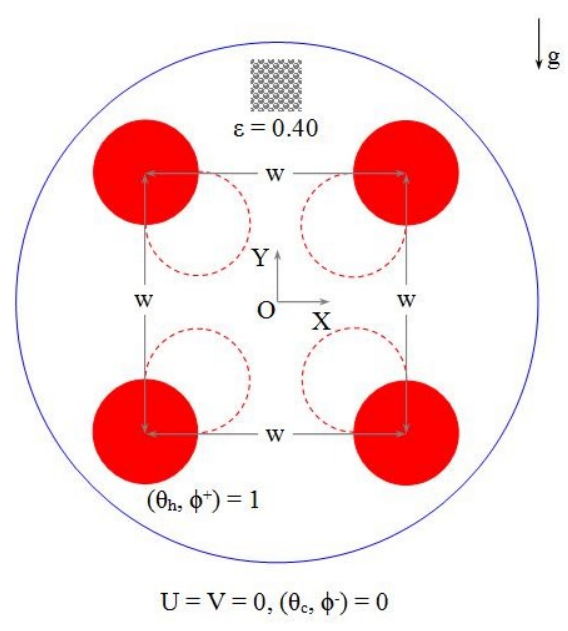

(Case 01)

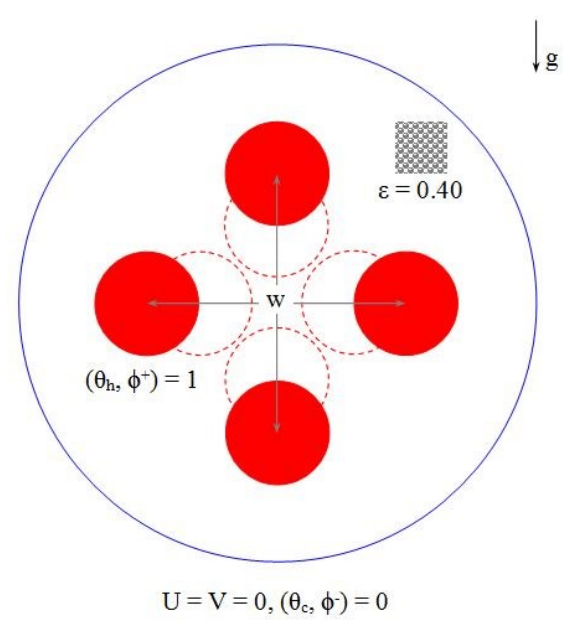

(Case 02)

Fig. 1 Simulation domain with its boundary conditions.

The dimensionless conservation equations describing the transport phenomenon inside the porous medium can be written in Cartesian coordinates as:

$$
\begin{aligned}
& \frac{\partial \mathrm{U}}{\partial \mathrm{X}}+\frac{\partial \mathrm{V}}{\partial \mathrm{Y}}=0 \\
& \frac{1}{\varepsilon^{2}}\left(\mathrm{U} \frac{\partial \mathrm{U}}{\partial \mathrm{X}}+\mathrm{V} \frac{\partial \mathrm{U}}{\partial \mathrm{Y}}\right)=-\frac{\partial \mathrm{P}}{\partial \mathrm{X}}-\frac{\operatorname{Pr}}{\mathrm{Da}} \mathrm{U}+\operatorname{Pr}\left(\frac{\partial^{2} \mathrm{U}}{\partial \mathrm{X}^{2}}+\frac{\partial^{2} \mathrm{U}}{\partial \mathrm{Y}^{2}}\right) \\
& \frac{1}{\varepsilon^{2}}\left(\mathrm{U} \frac{\partial \mathrm{V}}{\partial \mathrm{X}}+\mathrm{V} \frac{\partial \mathrm{V}}{\partial \mathrm{Y}}\right)=-\frac{\partial \mathrm{P}}{\partial \mathrm{Y}}-\frac{\operatorname{Pr}}{\mathrm{Da}} \mathrm{V}+\operatorname{Pr}\left(\frac{\partial^{2} \mathrm{~V}}{\partial \mathrm{X}^{2}}+\frac{\partial^{2} \mathrm{~V}}{\partial \mathrm{Y}^{2}}\right)+\operatorname{Ra} \operatorname{Pr}(\theta+\mathrm{N} \phi)
\end{aligned}
$$




$$
\begin{aligned}
& \mathrm{U} \frac{\partial \theta}{\partial \mathrm{X}}+\mathrm{V} \frac{\partial \theta}{\partial \mathrm{Y}}=\left(\frac{\partial^{2} \theta}{\partial \mathrm{X}^{2}}+\frac{\partial^{2} \theta}{\partial \mathrm{Y}^{2}}\right) \\
& \mathrm{U} \frac{\partial \phi}{\partial \mathrm{X}}+\mathrm{V} \frac{\partial \phi}{\partial \mathrm{Y}}=\frac{1}{\mathrm{Le}}\left(\frac{\partial^{2} \phi}{\partial \mathrm{X}^{2}}+\frac{\partial^{2} \phi}{\partial \mathrm{Y}^{2}}\right)
\end{aligned}
$$

where $\mathrm{Da}$ is the Darcy number, Le is the Lewis number, $\mathrm{N}$ is the buoyancy ratio, $\mathrm{Pr}$ and $\mathrm{Ra}$ are the Prandtl and the thermal Rayleigh numbers.

\section{NUMERICAL PROCEDURE}

The Cartesian Cut-Cell method, also called Cartesian Grid method [14], is used to handle the inner and the outer circular cylinders, (Fig. 2). The control volumes found into the inner ones are assumed to be inactive (i.e. $\mathrm{U}=\mathrm{V}=0$ ) following "Eq. (8)". The same is imposed to the outside part of the outer cylinder (see "Eq. (9)").

$$
\begin{aligned}
& (x-0.5)^{2}+(y-0.5)^{2} \leq\left(\frac{D_{i}}{2}\right)^{2} \\
& (x-0.5)^{2}+(y-0.5)^{2}>\left(\frac{D_{o}}{2}\right)^{2}
\end{aligned}
$$

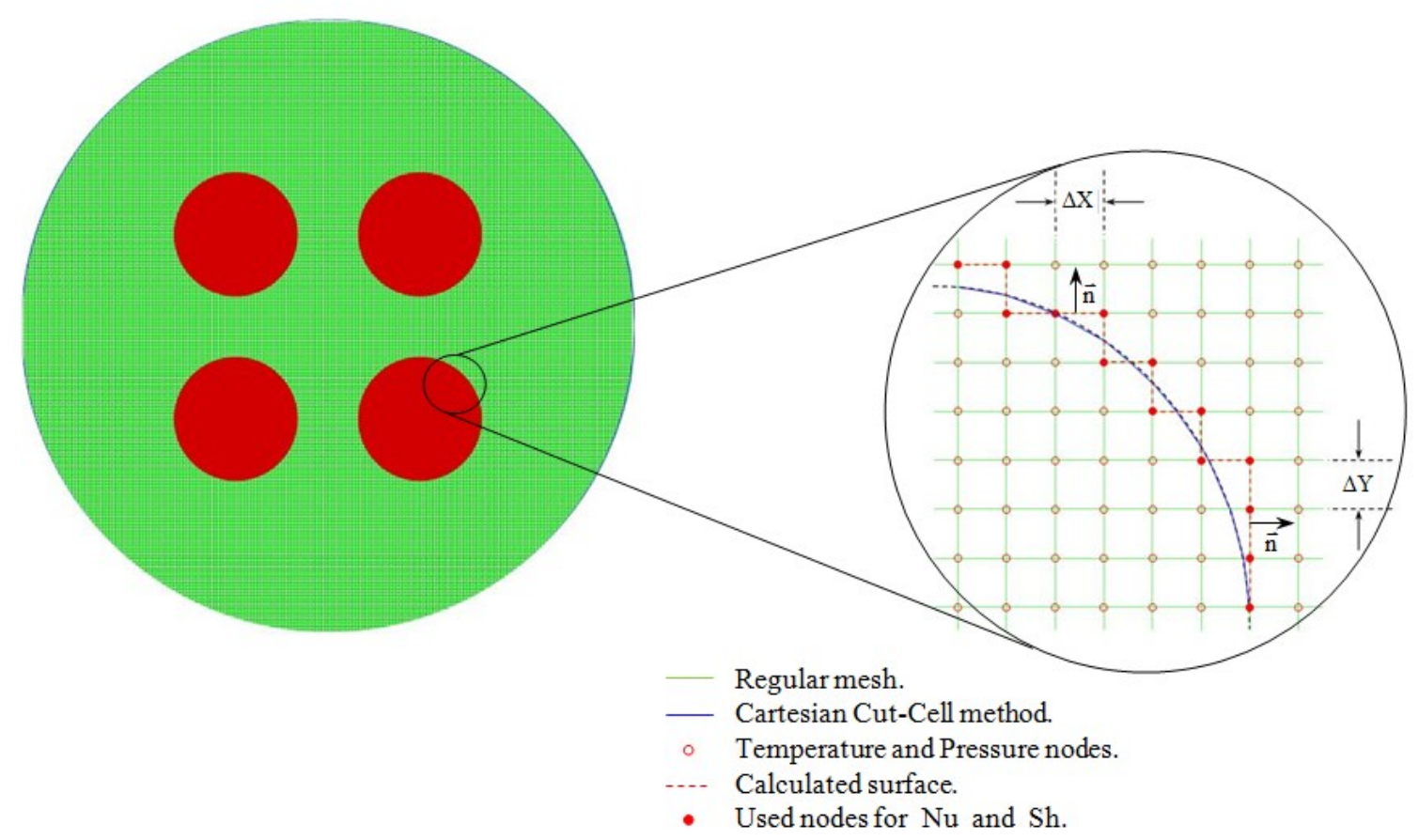

Fig. 2 Grid arrangement and Cartesian Cut-Cell Method.

The governing conservation equations are discretized in space using the finite volume approach, when the convection-diffusion terms were treated with a Power-Law scheme. The resulting algebraic equations, with the associated boundary conditions, are then solved using the line by line method. As the momentum equation is formulated in terms of the primitive variables (U, V and P), the iterative procedure includes a pressure correction calculation method, namely SIMPLER [15], to solve the pressure-velocity coupling. 
Noted that the convergence criterion for temperature, concentration, pressure, and velocity is given as:

$$
\frac{\sum_{j=1}^{m} \sum_{i=1}^{n}\left|\xi_{i, j}^{t+1}-\xi_{i, j}^{t}\right|}{\sum_{j=1}^{m} \sum_{i=1}^{n}\left|\xi_{i, j}^{t+1}\right|} \leq 10^{-5}
$$

where $\mathrm{m}$ and $\mathrm{n}$ are the numbers of grid points in $\mathrm{X}$ - and Y-directions, respectively. $\xi$ is any of the computed field variables, and $t$ is the iteration number.

Once the velocity, the temperature and the solutal fields are obtained, each cylinder surfaceaveraged Nusselt and Sherwood numbers can be computed using the following expressions:

$$
\begin{aligned}
& \mathrm{Nu}=\frac{\partial \theta}{\partial \mathrm{n}} \mid ; \mathrm{Nu}_{\mathrm{Avg}}=\frac{1}{\mathrm{~A}} \int_{0}^{\mathrm{A}} \mathrm{Nu} \mathrm{dS} \\
& \mathrm{Sh}=\frac{\partial \phi}{\partial \mathrm{n}} \mid ; \mathrm{Sh}_{\mathrm{Avg}}=\frac{1}{\mathrm{~A}} \int_{0}^{\mathrm{A}} \mathrm{Sh} \mathrm{dS}
\end{aligned}
$$

where $\mathrm{n}$ is the normal direction with respect to the inner or the outer surface (see for instance Fig. 2), and $\mathrm{A}$ is the surface area of each cylinder.

\section{CODE VALIDATION}

The performance of the using code via the double-diffusive natural convection problem into a confined porous medium is established by comparing predictions with other numerical results and experimental data, and by verifying the grid independence of the present results.

First, the present results are consistent with previous computations, namely those of Goyeau et al. [16] which deal with double-diffusive natural convection in a square porous enclosure. By taking into account the same hypotheses, Fig. 3 displays the comparison between the both predictions in term of Streamlines and Isotherms and Iso-concentration plots. Fig. 4 illustrates the Verticalvelocity computed along the mid-plane of the enclosure for various values of the buoyancy ratio, when Fig. 5 gives the mean Nusselt and Sherwood numbers, obtained by the present work and the previous ones [16]. As we can see, the present results and those of Goyeau et al. are in excellent agreement with a maximum discrepancy of about $2 \%$.

Then, to ascertain the numerical code validity with natural convection in horizontal cylindrical annulus, those obtained experimentally by Bishop et al. [17] have been taken into consideration. Fig. 6 shows the comparison between the previous experimental data and the present predictions. Once again, the numerical results exhibit a great qualitative concordance with the experimental ones, what support the use of our code to investigate the Thermosolutal phenomenon into such configurations. 

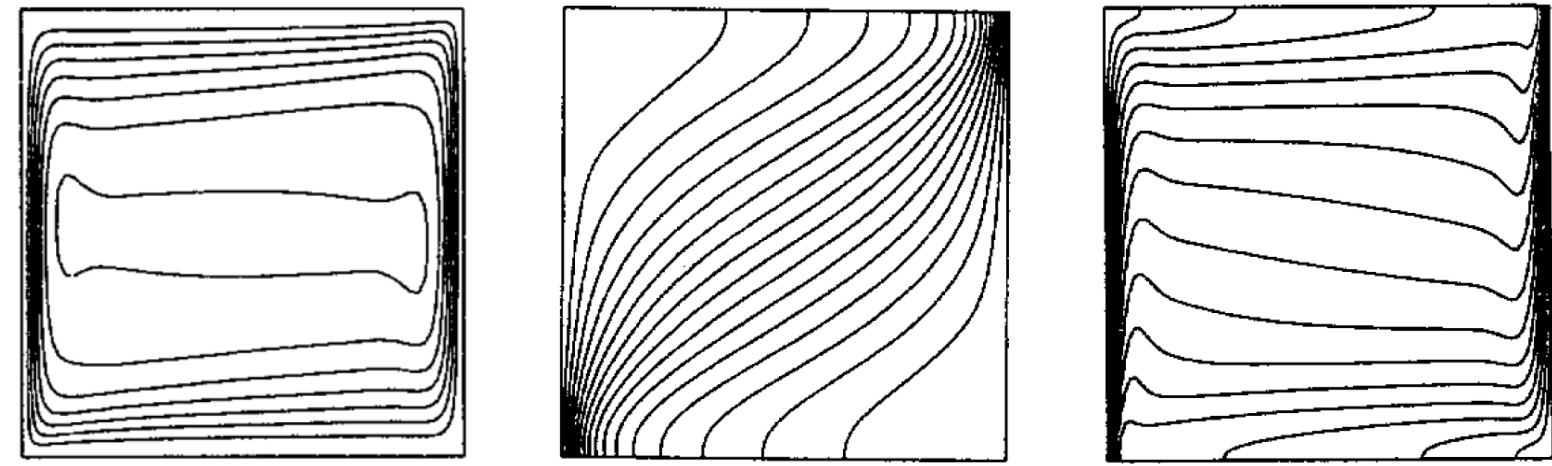

Goyeau et al. [16]
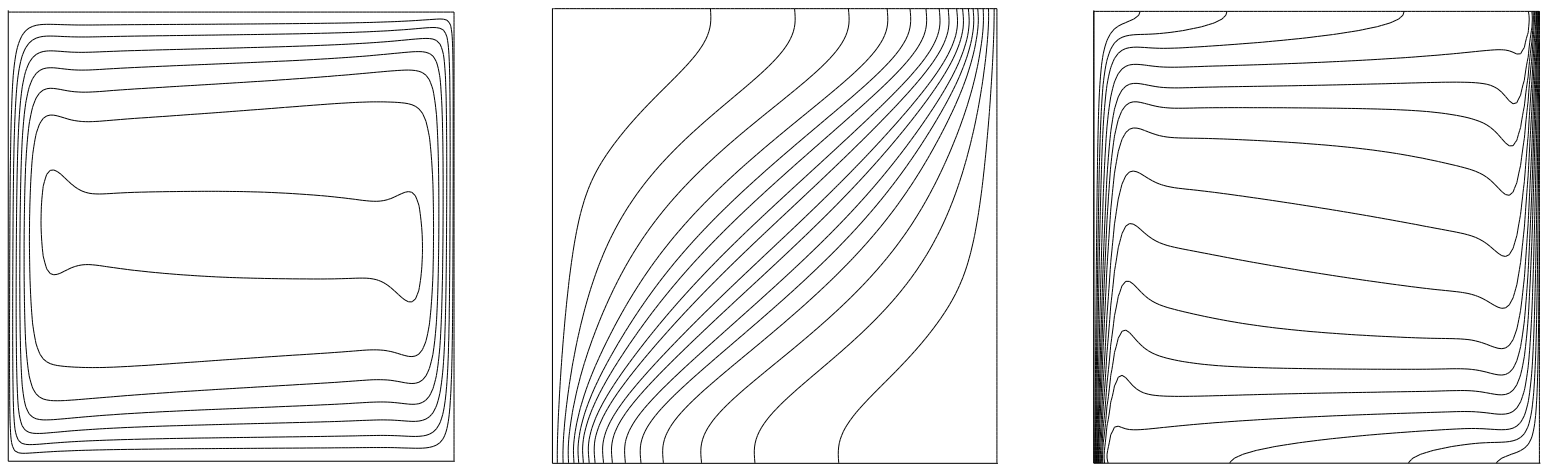

Present Predictions

(a)

(b)

(c)

Fig. 3 Streamlines (a), Isotherms (b) and Iso-concentrations (c). $\mathrm{Le}=10, \mathrm{~N}=10, \operatorname{Pr}=10, \mathrm{Ra}^{*}=10^{2}$.

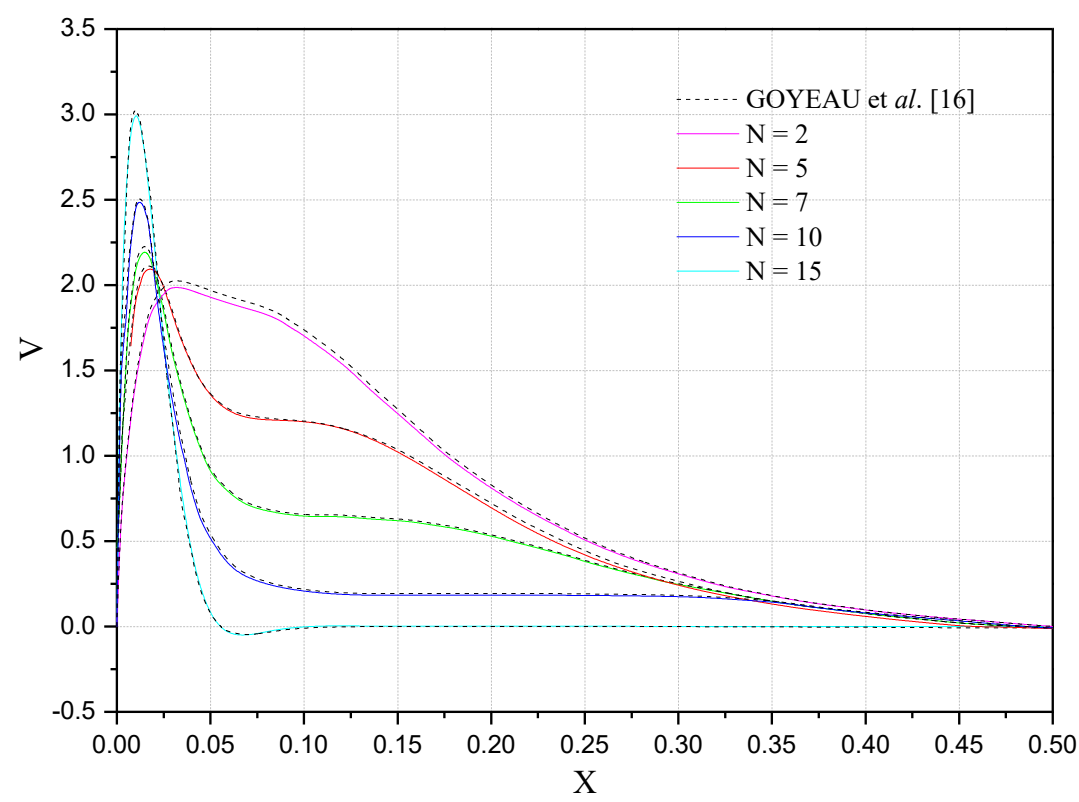

Fig. 4 Vertical-velocity profiles along the mid-plane of the enclosure, for various values of the buoyancy ratio. $\mathrm{Le}=100, \operatorname{Pr}=10, \mathrm{Ra}^{*}=10^{2}$. 

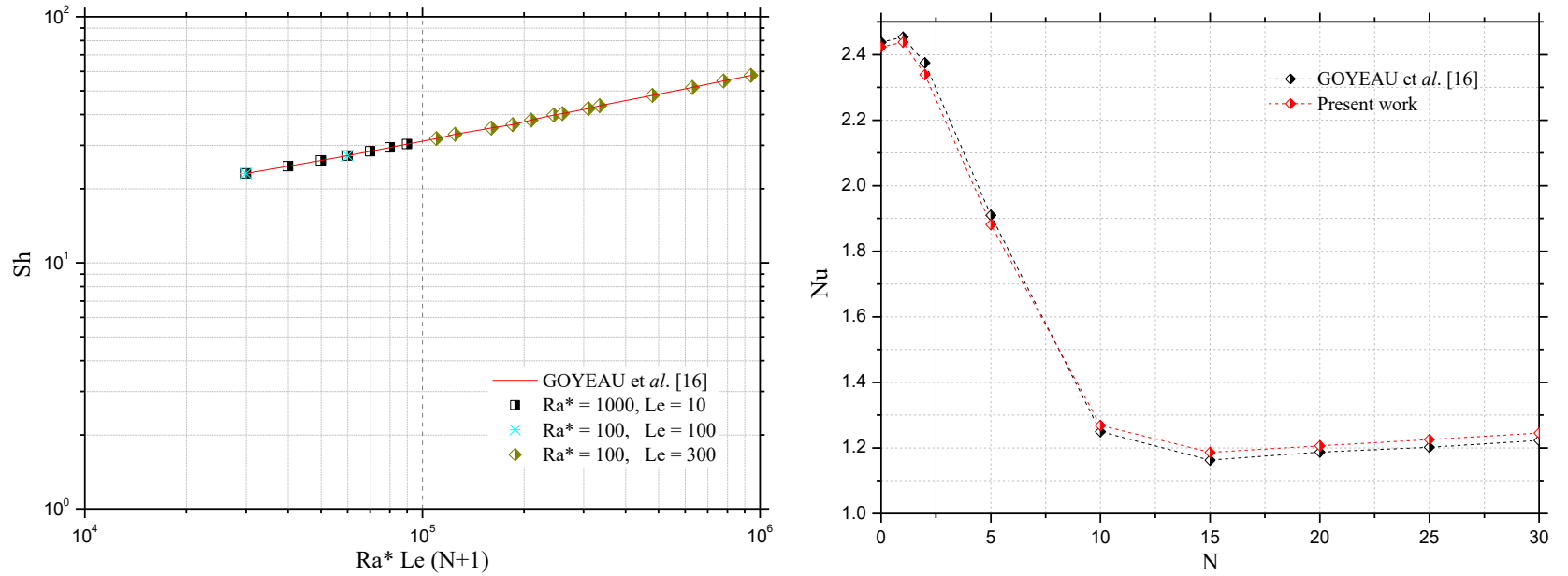

Fig. 5 Sherwood number function of the solutal Rayleigh Number (a),

Nusselt number into the porous square, $\mathrm{Le}=100, \mathrm{Ra}^{*}=100$ (b).

In order to determine a proper grid for the numerical simulations, a grid independence study is conducted for the double-diffusive natural convection into the partitioned circular cylinder previously shown in Fig. 1. Several mesh distributions ranging from $161^{2}$ to $401^{2}$ were tested and the mean Sherwood number, for the above uniform grids, is presented in Fig. 7. It is observed that a $201^{2}$ uniform grid is adequate for a grid independent solution. However, a fine structured mesh of $401^{2}$ is used to avoid round-off error for all other calculations in this investigation.
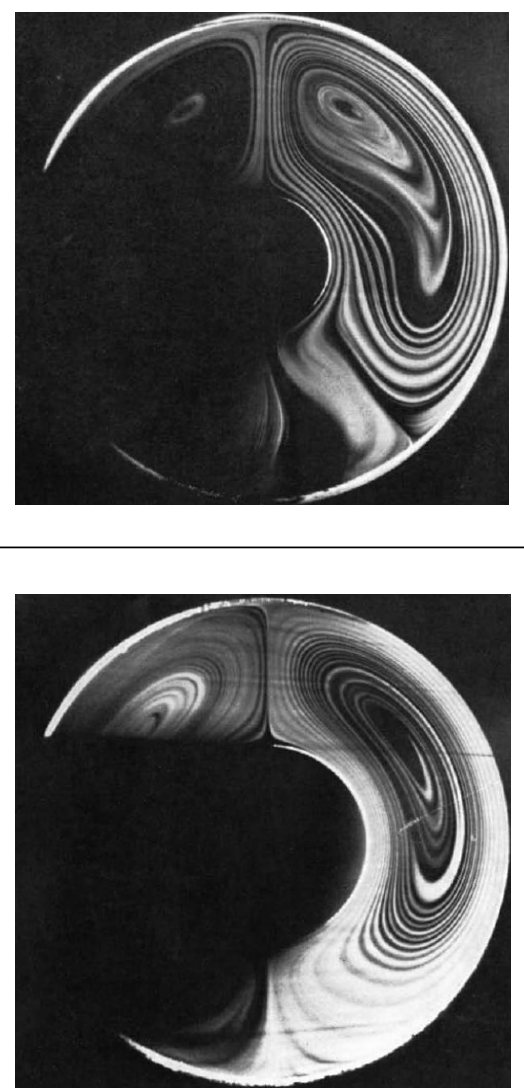

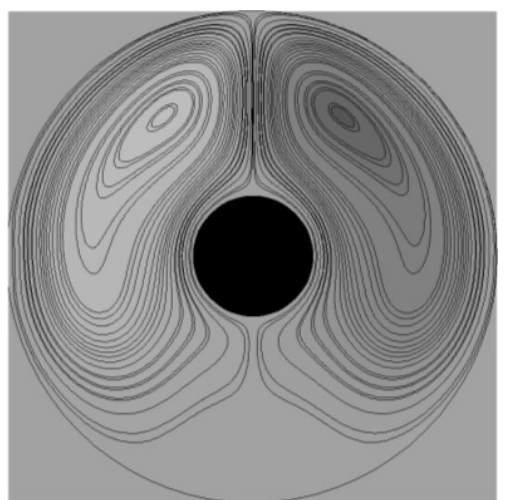

$\mathrm{D}_{\mathrm{i}}=0.24$

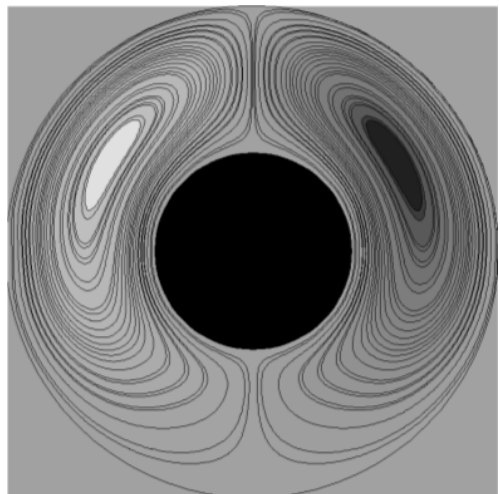

$\mathrm{D}_{\mathrm{i}}=0.40$

Bishop et al. [17]

Present Predictions

Fig. 6 Expérimental Streamlines validation, Bishop et al. [17]. 


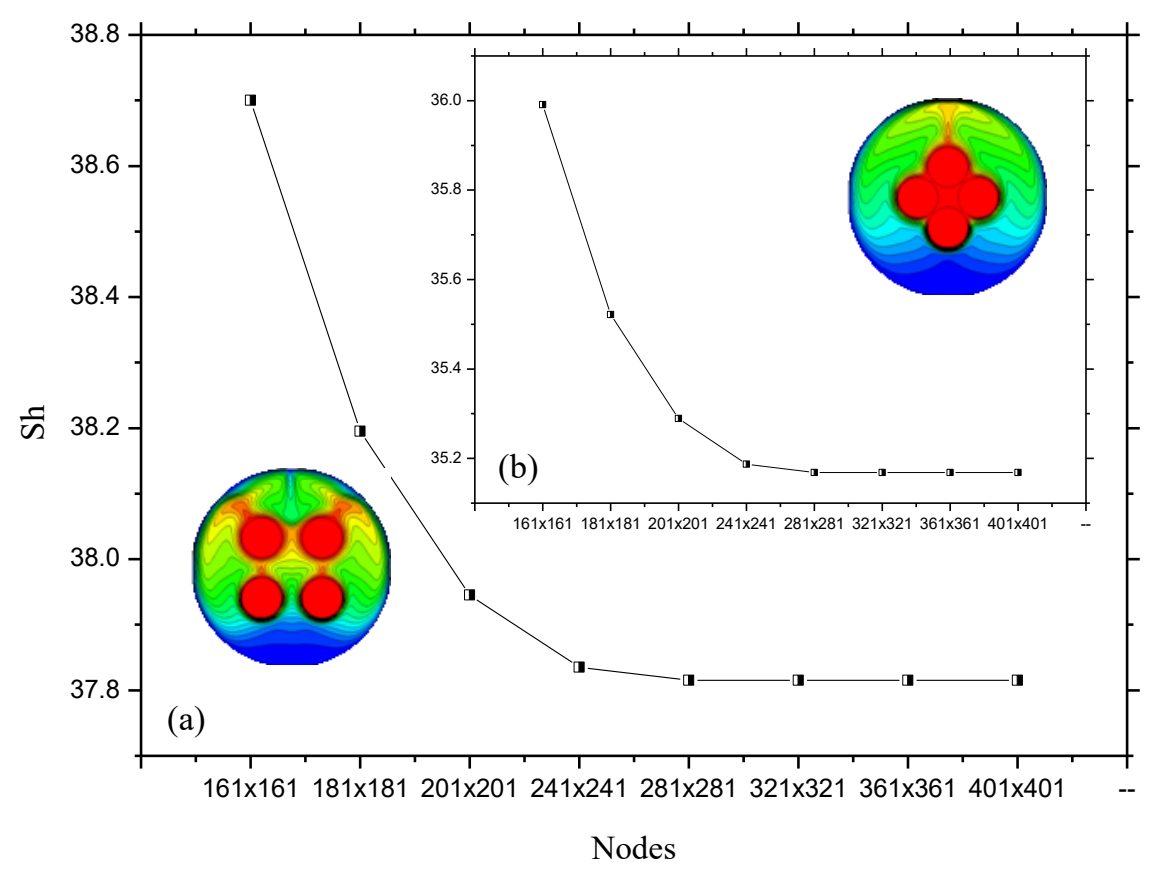

Fig. 7 Sherwood number of the porous circular cylinder for different uniform grids, Cylinders' rectangular arrangement (a), Cylinders' lozenge arrangement (b)

$$
\mathrm{Da}=10^{-4}, \mathrm{Le}=10, \mathrm{~N}=10, \mathrm{Ra}^{*}=100, \mathrm{w}=0.30 \text {. }
$$

\section{RESULTS AND DISCUSSION}

The range of parameters which have been examined in this study concerns the cooperating buoyancy forces domain. The value of the buoyancy ratio $\mathrm{N}$ has been taken from 0 to 30 and that, for various values of the porous thermal Rayleigh number $\mathrm{Ra}^{*}\left(=\mathrm{Ra} \mathrm{T}_{\mathrm{T}} \mathrm{Da}\right)$, ranging from 100 to 2000. The Lewis number has been taken between 10 and 300 when both the Prandtl and the Darcy numbers are fixed at 10 and $10^{-4}$, respectively. Noted that the cylinders aspect ratio $D_{i} / D_{0}$ is fixed as 0.20 , when the distance $\mathrm{w}$ between two each inner cylinders is ranging from 030 to 0.55 .

\section{Mass Transfer}

When the Lewis number Le and the cylinders' distance $w$ are fixed at 10 and 0.50 , respectively, the results illustrated in Fig. 8(a) have been obtained for two different values of the porous thermal Rayleigh number, 100 and 1000, and various values of the buoyancy ratio N.

In the both studied cases, mass transfer, defined using the mean Sherwood number, is found to be an increasing function of the buoyancy ratio $\mathrm{N}$ and the porous thermal Rayleigh number $\mathrm{Ra}^{*}$ as well. This behavior confirms the fact that the global buoyancy term in the momentum equation, $\mathrm{Ra}_{\mathrm{T}}$ $\operatorname{Pr}(\theta+N \phi)$, increases by increasing the thermal Rayleigh number and the buoyancy ratio, what enhancing the flow velocity and so the overall transfer.

By fixing the porous thermal Rayleigh number at 100, Fig. 8(b) shows the mean Sherwood number as a function of the buoyancy ratio $\mathrm{N}$, for two different values of the Lewis number, 30 and 300, respectively. The latter is perceived to increase with increases the Lewis number, for a given value of $\mathrm{N}$, due to increasing Schmidt number since the Prandtl number is already fixed at 10 . 


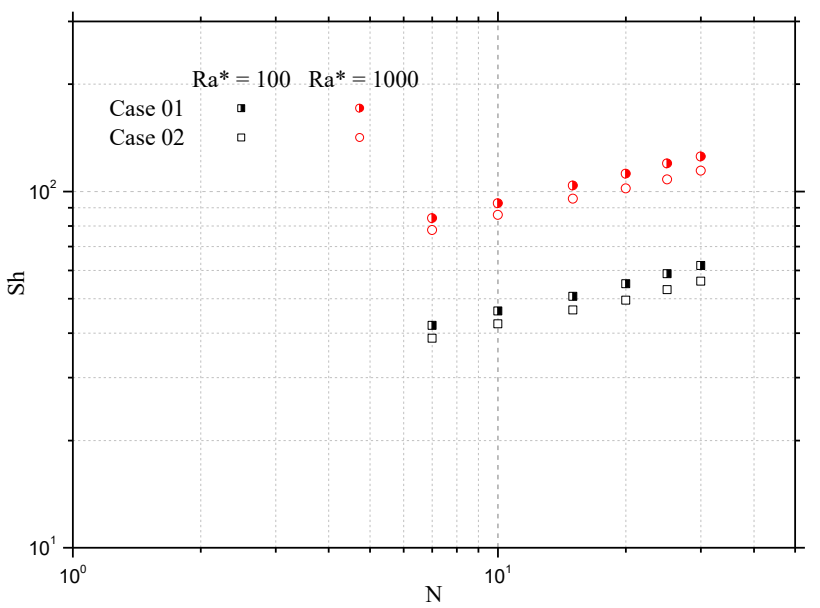

(a)

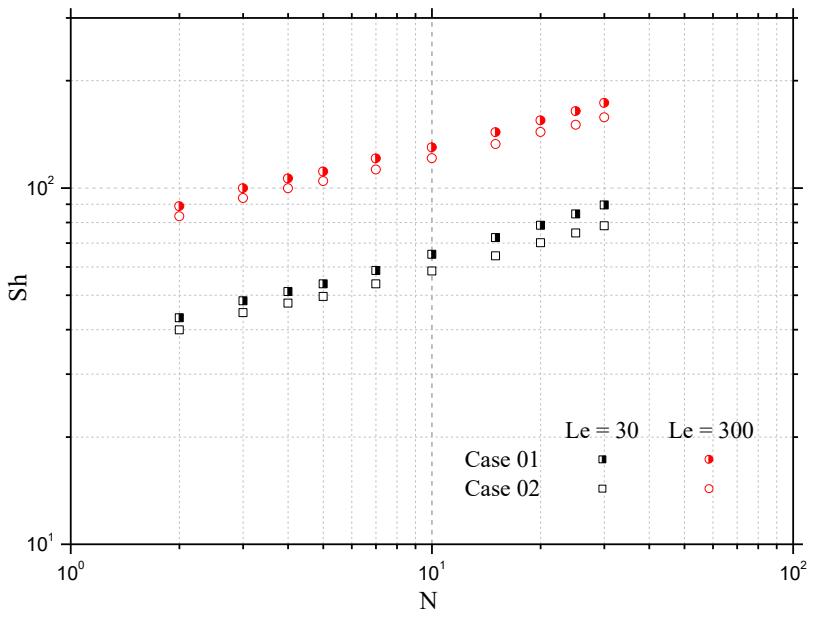

(b)

Fig. 8 Sherwood number of both cases as a function of the buoyancy ratio, $w=0.50$.

(a) $\mathrm{Le}=10, \mathrm{Ra}^{*}=100$ and 1000 .

(b) $\mathrm{Ra}^{*}=100, \mathrm{Le}=30$ and 300 .

Compared to the lozenge arrangement (Case 02), the mean Sherwood number of the rectangular arrangement (Case 01) is slightly greater, what makes the latter more desired.

Based on these observations, the numerical predictions obtained on the entire range of parameters given above may represent in Fig. 9 as a function of a general solutal Rayleigh Number $\mathrm{Ra}^{*}$ Le $(\mathrm{N}+1)$ and that, for various values of the cylinders' distance w. As we can see, the increase in the distance between the cylinders improves the overall Sherwood number and approves the Power-Law dependence of the mass transfer with the latter, what lead us eventually to the following general correlations:

$$
\begin{gathered}
\mathrm{Sh}_{\text {case } 01}=(0.92+3.09 \mathrm{w})\left[\frac{\mathrm{Ra} * \operatorname{Le}(\mathrm{N}+1)}{\mathrm{w}}\right]^{0.29} \\
\mathrm{Sh}_{\text {case } 02}=(0.86+2.88 \mathrm{w})\left[\frac{\mathrm{Ra} * \operatorname{Le}(\mathrm{N}+1)}{\mathrm{w}}\right]^{0.29} \\
\left(\mathrm{R}^{2}=0.991\right)
\end{gathered}
$$

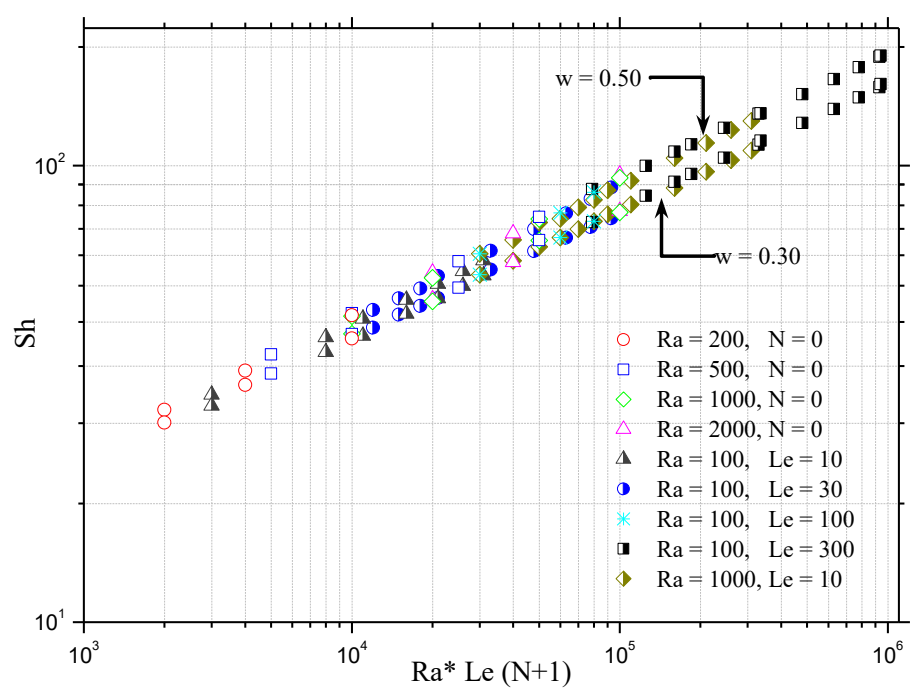

Fig 9 Sherwood number of Case 01 as a function of the solutal Rayleigh Number, for two values of the cylinders' distance w. 


\section{Heat Transfer}

About the heat transfer, Fig. 10 displays the impact of the buoyancy ratio and the Lewis number on the mean Nusselt number of the porous space. For both distances $\mathrm{w}=0.30$ and 0.50 , the mean transfer is found to be an increasing function of the buoyancy ratio and the cylinders' distance, when the latter decreases with increasing the Lewis number. This can be contributed to the thermal boundary layer thickness which is a function of $\mathrm{Le}^{1 / 2}[18]$.

Unlike the mass transfer, the mean Nusselt number calculated into the porous circular cylinder seems to be severely affected by the inner cylinders' arrangement. In fact, the difference computed between two values, of the same buoyancy ratio value, is found between $26 \%$ and $36 \%$, respectively, compared to the Sherwood number which is between $5 \%$ and $11 \%$.

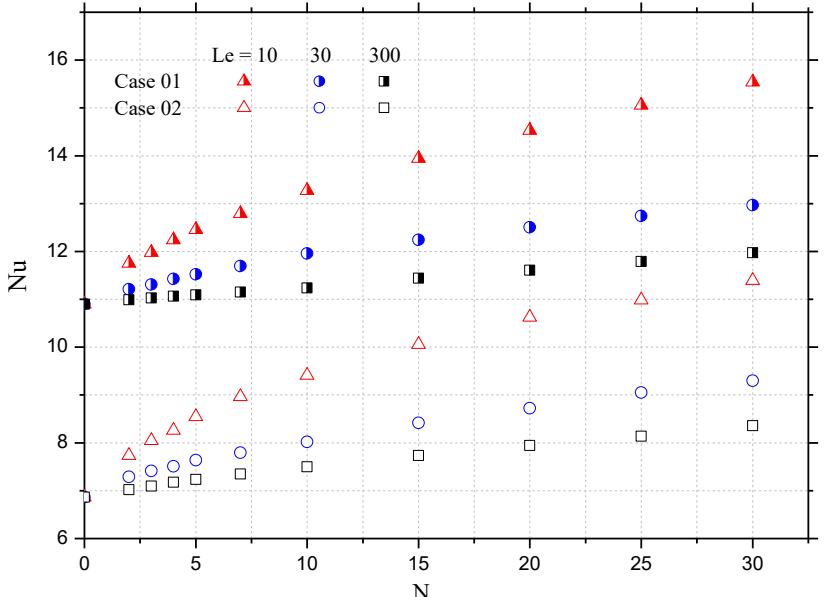

(a)

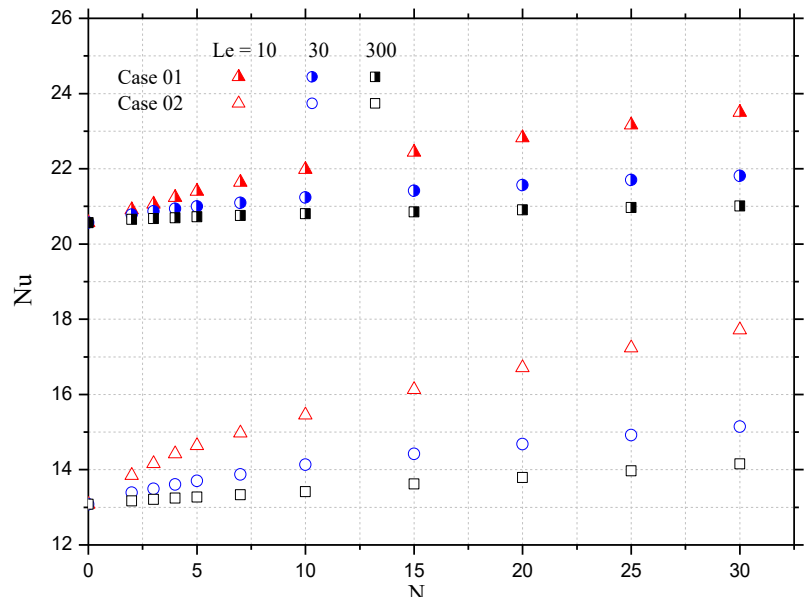

(b)

Fig 10 Mean Nusselt number of the both cases as a function of $\mathrm{N}$ and Le.

$$
\mathrm{Ra}^{*}=100, \mathrm{w}=0.30(\mathbf{a}), \mathrm{w}=0.50 \text { (b). }
$$

Taking into account the preceding results, correlating predictions of the heat transfer is done successfully using a dimensionless group $\left[\left(\mathrm{Ra}^{*} \mathrm{~N}(\mathrm{w})^{2.8}\right) / \mathrm{Le}\right]^{0.48}$ as shown Fig. 11 .

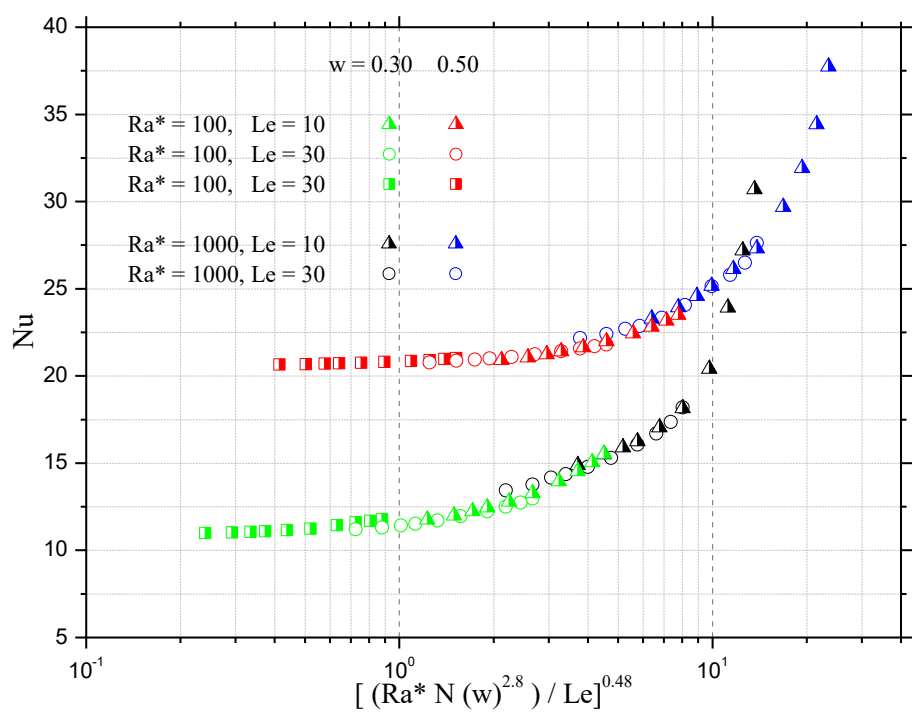

Fig. 11 Mean heat transfer Case 01 as a function of the dimensionless group $\left[\left(\mathrm{Ra}^{*} \mathrm{~N}(\mathrm{w})^{2.8}\right) / \mathrm{Le}\right]^{0.48}$.

Equations 14 and 15, give the mean heat transfer as a function of the pertinent parameters (Le, $\mathrm{N}, \mathrm{Ra}^{*}$ and $\mathrm{w}$ ) for both cases, Case 01 and 02, respectively: 


$$
\begin{array}{r}
\left.\mathrm{Nu}\right|_{\text {case } 01}=(38 \mathrm{w}-3.34)+(1.5-1.9 \mathrm{w})\left(\frac{\mathrm{Ra}^{*} \mathrm{~N}}{\mathrm{Le}}(\mathrm{W})^{2.8}\right)^{0.576} \\
\left(\mathrm{R}^{2}=0.987\right) \\
\left.\mathrm{Nu}\right|_{\text {case } 02}=(30 \mathrm{w}-6.11)+(1.94-2.3 \mathrm{w})\left(\frac{\mathrm{Ra}^{*} \mathrm{~N}}{\mathrm{Le}}(\mathrm{W})^{2.8}\right)^{0.50} \\
\left(\mathrm{R}^{2}=0.981\right)
\end{array}
$$

The latter are available for a Lewis number ranging from 1 to 300 , a buoyancy ratio $\mathrm{N}$ taking between 0 and 30, a porous thermal Rayleigh number in between 100 and 1000, and a distance w in between 0.30 and 0.55 .

\section{CONCLUSION}

The analysis of Thermosolutal natural convection into an horizontal Darcy-Brinkman porous circular cylinder having four inner heat and solute cylinders, arranged in a rectangular or lozenge manner, was numerically realized through this paper. In general and unlike the mass transfer, the mean heat transfer seems to be severely affected by the cylinders' arrangement. Then, the use of rectangular disposition is found more desired for optimal transfer.

By taking into account the effect of pertinent parameters such the Lewis and porous thermal Rayleigh numbers, the buoyancy ratio and the distance in between two successive cylinders as well, new prediction equations "Eqs. 12-15" were obtained that give the mean heat and mass transfers into such geometry. This correlation may count as a complement to previous researches, and might prove particularly useful in verifying any instability analysis which might be put forth in the future.

\section{References}

[1] D.A. Nield, A. Bejan, Convection in porous media, Springer, Berlin, 1992.

[2] M. Mamou, P. Vasseur, E. Bilgen, Multiple solution for double-diffusive convection in a vertical porous enclosure, Int. J. Heat Mass Transf. 36 (1993) 2479-2498.

[3] P. Nithiarasu, K.N. Seetharamo, T. Sundarajan, Double-diffusive natural convection in an enclosure filled with fluid-saturated porous medium: A generalized Non-Darcy approach, Num. Heat Transf. A. 30 (1996) 413-426,.

[4] S. Ostrach, Natural convection with combined driving forces, Phys-Chem. Hydrodynamic 01 (1980) 233-247.

[5] Y. Kamotani, L.W. Wang, S. Ostrach, H.D. Jiang, Experimental study of natural convection in shallow enclosures with horizontal temperature and concentration gradients, Int. J. Heat Mass Transf. 28 (1985) 165-173.

[6] J. Lee, M.T. Hyun, K.W. Kim, Natural convection in confined fluids with combined horizontal temperature and concentration gradients, Int. J. Heat Mass Transf. 31 (1988) 1969-1977.

[7] C. Benard, D. Gobin, J. Thevenin, Thermosolutale natural convection in a rectangular enclosure: Numerical Results, in Heat Transfer in Convective Flows, ASME, New York (1989) 249-254.

[8] H. Han, T.H. Kuehn, A numerical simulation of double diffusive natural convection in a vertical rectangular enclosure, in Heat Transfer in Convective Flows, ASME, R.K. Shah, Ed. New York (1989) 149-154. 
[9] J. Chang, T.F. Lin, Unsteady thermosolutal opposing convection of liquid-water mixture in a square cavity- II: Flow structure and fluctuation analysis, Int. J. Heat Mass Transf. 36 (1993) 1333-1345.

[10]F. Chen, Double-diffusive fingering convection in a porous medium, Int. J. Heat Mass Transf. 36 (1993) 793-807.

[11] O. Trevisan, A. Bejan, Heat and mass transfer by high Rayleigh number convection in a porous medium heated from below, Int. J. Heat Mass Transf. 30 (1987) 2341-2356.

[12] T.F. Lin, C.C. Huang, T.S. Chang, Transient binary mixture natural convection in a square enclosure, Int. J. Heat Mass Transf. 33 (1990) 287-299.

[13]R. Bennacer, Thermosolutal convection: fluid flow and heat transfer numerical simulations, Ph.D. Thesis, Pierre and Marie Curie, Paris (1993).

[14]P. Colella, D.T. Graves, B.J. Keen, and D. Modiano. A cartesian grid embedded boundary method for hyperbolic conservation laws. J. Comput. Phys., 211(1) (2006) 347-366.

[15] S.V. Patankar, Numerical heat transfer and fluid flow, Mc Grow, New York (1980).

[16] B. Goyeau, J.P. Songbe, D. Gobin, Numerical study of double-diffusive natural convection in a porous cavity using the Darcy-Brinkman formulation, Int. J. Heat Mass Transf. 39(7) (1996) 1363-1378.

[17]E.H. Bishop, C.T. Carleyt and R.E. Powe, Natural convective oscillatory flow in cylindrical annuli, Int. J. Heat Mass Transf. 11 (1968) 1741-1752.

[18] O. Trevisan, A. Bejan, Natural convection with combined heat and mass transfer buoyancy effects in a porous medium, Int. J. Heat Mass Transf. 28 (1985) 1597-1611. 